\title{
Adult Case of Parachute-Like Asymmetrical Mitral Valve Complicated by Mitral Stenosis and Thrombus Formation
}

Yujiro Kawai, MD; Takahito Yokoyama, MD; Gentaku Hama, MD;

Yasuyuki Toyoda, MD; Miki Horigome, MD, PhD; Yasutoshi Tsuda, MD, PhD;

Yoshikazu Yazaki, MD, PhD; Takahiro Takemura, MD, PhD
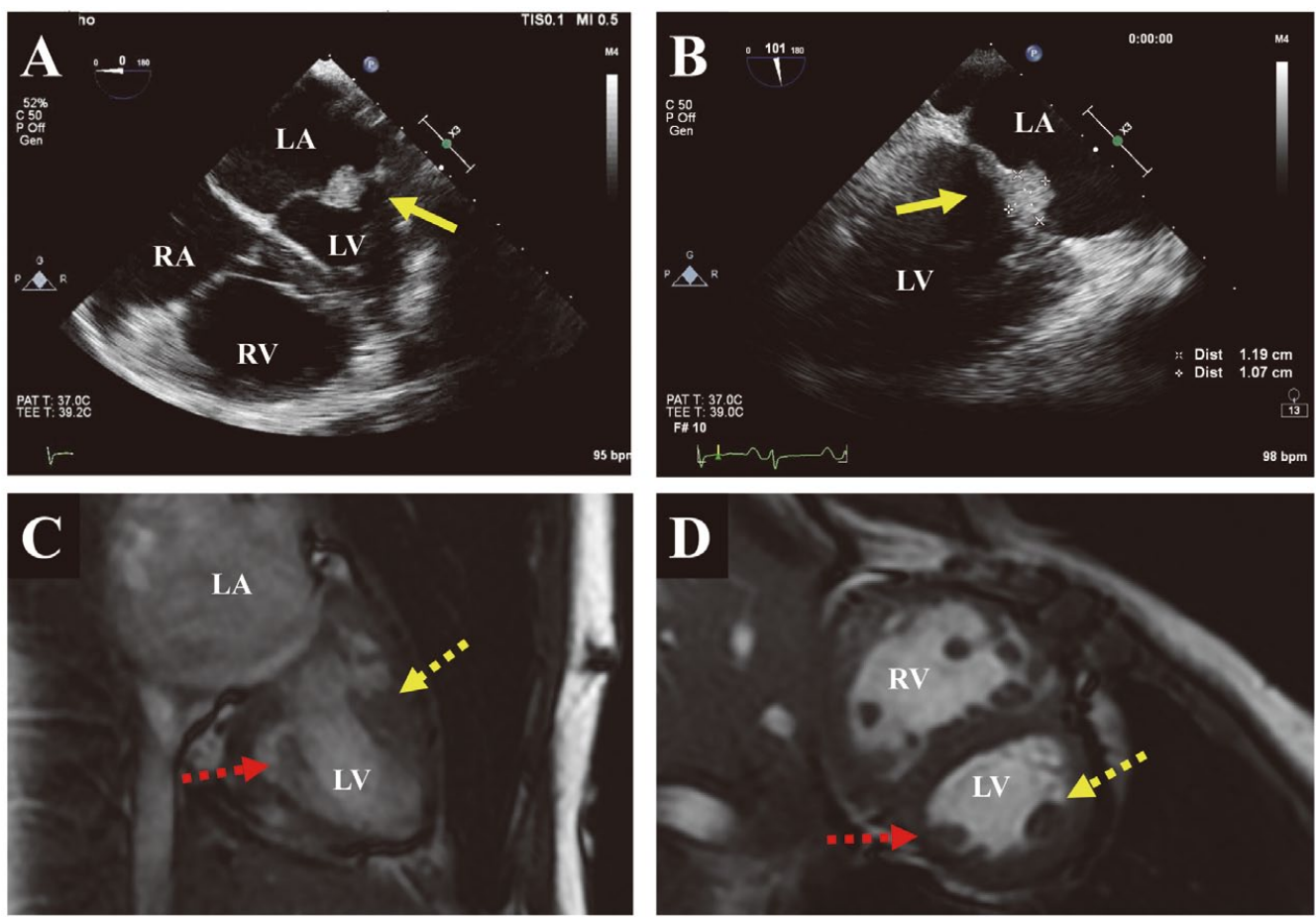

Figure 1. (A) Four-chamber and (B) 2-chamber transesophageal echocardiography showing a hyperechoic mass (12×11 mm; yellow arrows). (C) Two-chamber and (D) short axis magnetic resonance imaging showing two similarly sized papillary muscles, namely, an anterolateral papillary muscle (dotted yellow arrows) and a posteromedial papillary muscle (dotted red arrows). LA, left atrium; LV, left ventricle; RA, right atrium; RV, right ventricle.

A previously healthy 33-year-old man was admitted to Saku Central Hospital Advanced Care Center with suspected infective endocarditis. He had a 2-month history of dyspnea on exertion and cough. Transthoracic and transesophageal echocardiography showed severe mitral valve stenosis with a mitral valve mass measuring $12 \times 11 \mathrm{~mm}$ attached to the posterior leaflet
(Figure 1A,B). Mitral valve mean pressure gradient was elevated to $16 \mathrm{mmHg}$ and MVA was reduced to around $1.0 \mathrm{~cm}^{2}$. The orifice of the mitral valve was located in the lateral site, and both anterior and posterior commissures were fused and unclear. There was also mild tricuspid valve regurgitation, but no other valve stenosis, regurgitation, septal defect, hypoplastic left heart, or abnormal structure

Received February 26, 2017; revised manuscript received July 2, 2017; accepted July 13, 2017; released online August 11, 2017 Time for primary review: 36 days

Department of Cardiovascular Surgery (Y.K., T.Y., G.H., Y. Toyoda, Y. Tsuda, T.T.), Department of Cardiology (M.H., Y.Y.), Saku Central Hospital Advanced Care Center, Saku, Japan

Mailing address: Yujiro Kawai, MD, Department of Cardiovascular Surgery, Saku Central Hospital Advanced Care Center, 3400-28 Nakagomi, Saku 385-0393, Japan. E-mail: yujiro.roadster@gmail.com

ISSN-1346-9843 All rights are reserved to the Japanese Circulation Society. For permissions, please e-mail: cj@j-circ.or.jp 


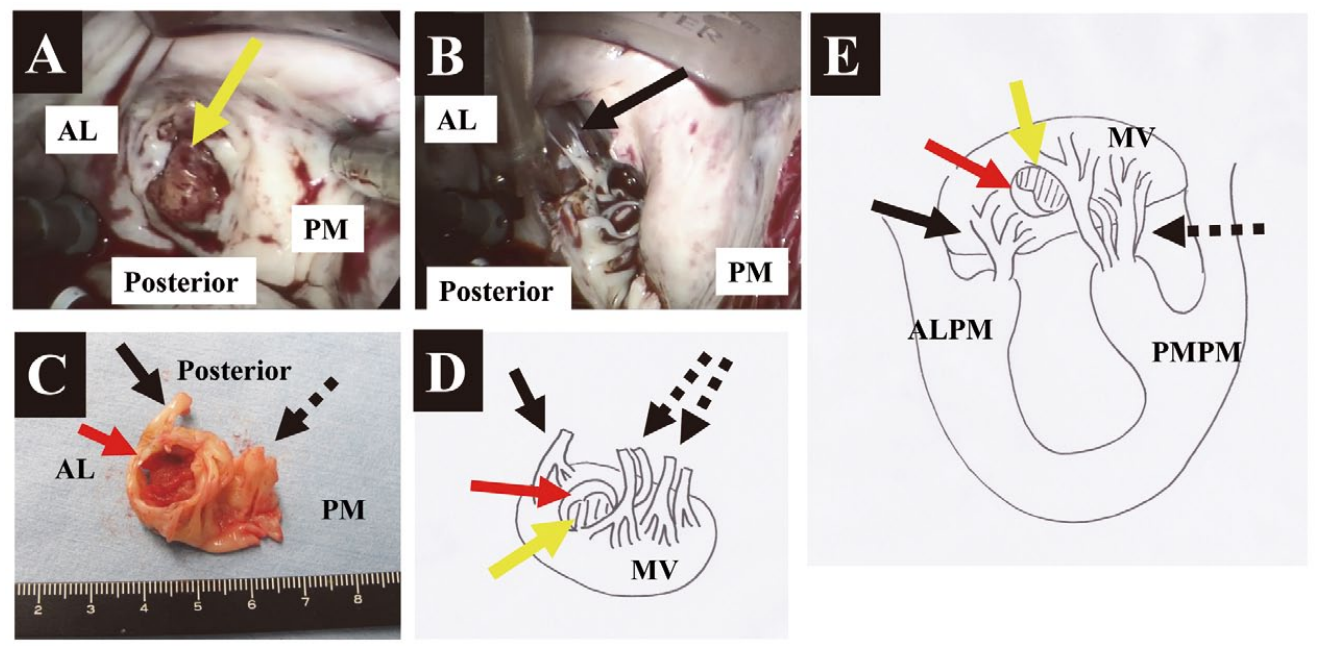

Figure 2. (A,B) Intraoperative findings. (C,D) Resection of mitral valve (MV) and the corresponding schematic diagram. (E) Schematic diagram of the relationship between the MV, chords and papillary muscles. (A,C,D,E) Both anterior and posterior commissures of the MV were fused and a small orifice was opened at the anterolateral side of the leaflet (red arrow); the thrombus was attached to this orifice (yellow arrows). (B,D,E) Anterolateral papillary muscle ended just below the MV annulus and short chords were attached to the leaflet (black arrow). (C,D,E) Chords were attached to the leaflet asymmetrically (black arrow and black dotted arrows). AL, anterolateral; ALPM, anterolateral papillary muscle; PM, posteromedial; PMPM, posteromedial papillary muscle.

in the heart. Although blood cultures were negative, the mass was suspected to be a thrombus or tumor. Electrocardiogram indicated normal sinus rhythm with a heart rate of 88 beats/min. Magnetic resonance imaging (MRI) showed a mass attached to the mitral valve and two papillary muscles of similar size (Figure 1C,D). MRI indicated no other structural abnormalities in the left ventricle. Computed tomography showed no abnormalities in the aorta such as coarctation of the aorta.

The patient underwent mitral valve replacement via left mini thoracotomy and was implanted with a $29-\mathrm{mm}$ ATS mechanical mitral prosthesis. During the operation, the mitral valve mass was confirmed to be a thrombus. Both anterior and posterior commissures of the mitral valve were fused and a small orifice was opened at the lateral side of the leaflet (Figure 2A,B). Most of the chordae were attached to the posteromedial papillary muscle and small chords were attached to the anterolateral papillary muscle. The anterolateral papillary muscle was ended just below the mitral valve annulus and its chords were shorter than those attached to the posteromedial papillary muscles (Figure 2C,D). From these findings, we concluded that the patient had congenital parachute-like asymmetrical mitral valve (PLAMV).

Parachute mitral valve was originally defined as having all chords attached to one papillary muscle. There is also a different clinical entity, characterized by two papillary muscles with a focalized attachment of the chordae into the dominant muscle, which is distinguished from parachute mitral valve, and is known as PLAMV. ${ }^{1}$ Isolated congenital mitral valve stenosis caused by PLAMV is rare because it is often associated with other congenital heart diseases. ${ }^{2,3}$
There has been one other reported case of infective endocarditis associated with PLAMV, ${ }^{4}$ but this is the first case of PLAMV complicated by thrombus formation. Because the present mitral valve stenosis had been mild during childhood, the patient had been asymptomatic and had never been diagnosed with heart murmur or any other physical findings. Thrombus formation occurred in the left atrium (LA) as a result of LA stasis due to mitral valve stenosis, and might have led to severe mitral stenosis and symptomatic heart failure. ${ }^{5,6}$

\section{Disclosures}

The authors declare no conflict of interest.

\section{References}

1. Oosthoek PW, Wenink AC, Macedo AJ, Gittenberger-de Groot AC. The parachute-like asymmetrical mitral valve and its papillary muscles. J Thorac Cardiovasc Surg 1997; 114: 9-15.

2. Collins-Nakai RL, Rosenthal A, Castaneda AR, Bernhard WF, Nadas AS. Congenital mitral stenosis: A review of 20 years' experience. Circulation 1997; 56: 1039-1047.

3. Moore P, Adatia I, Spevak PJ, Keane JF, Perry SB, Castaneda $\mathrm{AR}$, et al. Severe congenital mitral stenosis in infants. Circulation 1994; 89: 2099-2106.

4. Showkathali R, Birdi I, Khokhar A. Infective endocarditis in a parachute-like asymmetrical mitral valve. Eur $J$ Echocardiogr 2009; 10: 476-478.

5. Agmon Y, Khandheria BK, Getile F, Seward JB. Clinical and echocardiographic characteristics of patients with left atrial thrombus and sinus rhythm. Circulation 2002; 105: $27-31$.

6. Kasliwal RR, Mittal S, Kanojia A, Singh RP, Prakash O, Bhatia $\mathrm{ML}$, et al. A study of spontaneous echo contrast in patients with rheumatic mitral stenosis and normal sinus rhythm: An Indian perspective. Br Heart J 1995; 74: 296-299. 